\title{
Theory and Practice of Modeling and Simulations in Programs for engeneering Education
}

\author{
Josef Sedivy \\ University of Hradec Kralove, Faculty of Science, Department of Informatics, Rokitanskeho 62, 500 \\ 03 Hradec Kralove, Czech Republic \\ josef.sedivy@uhk.cz
}

Keywords: Scientific modelling, Technical modelling, Parametric modelling, Simulation.

\begin{abstract}
Procedures applied in the current industrial engineering practice put new demands on knowledge and skills of graduates of technical schools, connected with a significant proportion of using of tools for design, simulation, production technology and data management solutions for the design and technological tasks. Computer-aided engineering activities open up new approaches to creation and analysis of designs and also for creation of processes of manufacturing and assembly. A large number of data sets, the complexity of procedures and the need of sharing of information in various points on the planet with adequate check and security of shared information required the deployment of data management systems and processes.
\end{abstract}

\section{Introduction}

Today, programs for technical modelling used in almost every field or industry. We see them in technical industries as well as in our everyday life and even in areas where we would never expect to see them. It generally replaces the creativity and imagination of the designers, and in many cases it helps determine collision situations and points during the creation of new products. Programs for technical modelling we used in almost every field or industry. We see them in technical industries as well as in our everyday life and even in areas where we would never expect to see them. It generally replaces the creativity and imagination of the designers, and in many cases it helps determine collision situations and points during the creation of new products.

\section{Scientific modelling}

Modelling we use not only for solving problems of practice. As in this article we solve computer modelling of virtual prototypes. Scientific modelling is intended also to perform various studies and experiments or simulations of phenomena and processes. It is important to note, however, that the model can in no way cover the actual reality, but a simplified view on a certain part of the real world [1]. For the successful creation and exploitation of the model is necessary to understand the problem we want to model, capture, have a pre-specified target and simultaneously use a good quality source data. Modelling is a multidisciplinary activity, since it can contribute to knowledge of mathematics and physics, systems theory, probability theory, computer science, cybernetics and cognitive science, operations research and others. In practice, we encounter a great variety of different types of models [2]. Only some of them can be understood as models suitable for use in scientific work. Models can be categorized according to the following criteria [3]:

- $\quad$ Semantics - what the model represents and acts as such;

- Ontology - what means models can be heard;

- $\quad$ Gnoseology - both models used to develop knowledge;

- $\quad$ philosophy - both models reflect reality, what are the implications of the model approach to science and reality, both models can explain by natural laws. 


\section{Application in parametric modelling}

Parametric modellers may use different environments and user interfaces, but the general principles of parametric modelling are similar. The relevant software application is mostly used as another creative tool. Models are created through sketching and through volume or spatial constructional elements. They must be clearly defined, without any redundant or contradictory information. There are always several solutions.

For practical example we describe with mathemacic tools body. We use concepts such as topological spaces and topological views and $n$-manifold in $E m$. Topological space is a set of subsets $X$ with $\tau$. System $\tau$ must satisfy axioms:

1) $\emptyset \in \tau, X \in \tau$,

2) the intersection of a finite number of sets of $\tau$ must be back in $\tau$

3 ) the union of any number of sets (and innumerable) of $\tau$ is $\tau$ again. The system sets $\tau$ must be closed to the final penetration and any sum (even with the countless number of elements). File name the topology $\tau$ on $X$. The sets in $\tau$ are open sets and their accessories in $X$ closed sets. Topological space is denoted $(X, \tau)$.

Subset $U \subset X$ topological space $(X, \tau)$ is a neighborhood $p$ point, when there is an element $O \in \tau$, so that $p \in O$ and apply $O \subset U$. Neighborhood of a point will be denoted $p U(p)$. View $f$ topological space $(X, \tau)$ into a topological space $\left(X^{\prime}, \tau^{\prime}\right)$ is continuous if each image in the neighborhood of $p(X$, $\tau)$ is at the same time point $f$ surroundings $(p)$ in $\left(X^{\prime}, \tau^{\prime}\right)$. If $f$ is a bijection and $f f-1$ are continuous maps, then called $f$ "homeomorphism", a topological view. Two topological spaces are topologically equivalent if there is a homeomorphism between them. Homeomorphism is mutually unambiguous mapping between topological spaces that preserves the topological properties. In terms of topology, these two rooms the same properties are the same. Homeomorphism for the definition of an essential element.

In describing the body and its boundaries, we introduce the notion of manifold- $n E m N$-manifold in $E m$ where $m=n$, such $E m$ subset which is homeomorphic with $E n$. It is a manifold without boundary. $N$-in manifold with boundary $E m$ is then $E m$ subset which is homeomorphic with a positive half-space $E n+=\{(x 1, . ., x n) \in E n \mid x 1=0\}$. In practical implementation modeller to check the calculations correct topological elements. To check you can use Euler's formula, this is a necessary condition for correctness. Formula says that the number of walls, edges and vertices is given by the body. We solve first the bodies that have only one surface does not pass by them any holes (Fig. 1). They do not contain any internal cavity and the wall is homeomorphic with a circle. Such elements are topologically simple. They are all homeomorphic to the ball. When we describe $V$ number of vertices, edges $E$ number, number of walls $F$. You can then Euler's formula for this element can be written as:

$V \cdot F+2=E$

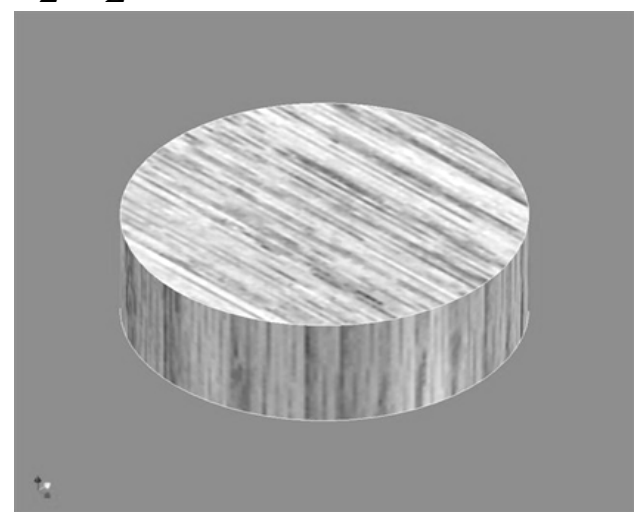

Fig. 1 Simplest element (body) with one surface

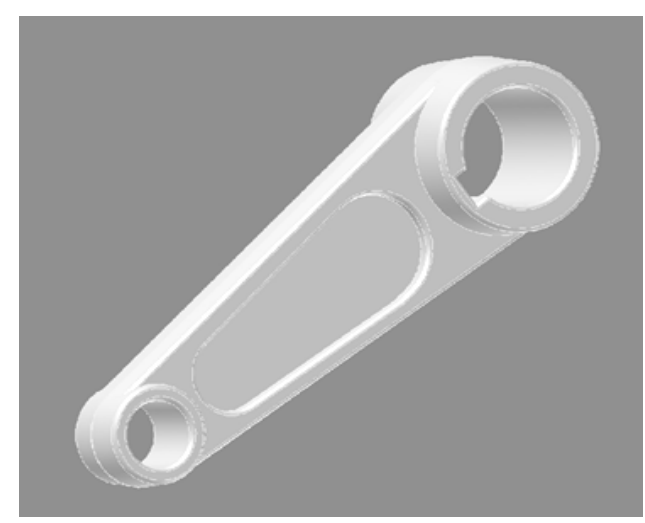

Fig. 2 Example of real technical body with the whole (not the internal cavity)

Body on fig. 2 is body with a whole (not the internal cavity) is bounded by a single continuous surface, while each wall element is homeomorphic with a circle. This mass is homeomorphic with a 
sphere with a certain number of closed units. Number of holes is a genus. For the body whose genus is $G$. The Euler formula here is:

$V+F=E+2(1-G)$

For housing with an internal cavity, the body is bounded by several separate closed surfaces. Denote the number of surfaces $S$. It is not necessary that every body panel is homeomorphic with a circle. Each wall is bounded by a single loop edges. $L$ the number of loops on surfaces of solids $G$ genes and the sum of the individual surfaces. Euler's formula has the shape:

$V \bullet F=E+(L-F)+2(S-G)$.

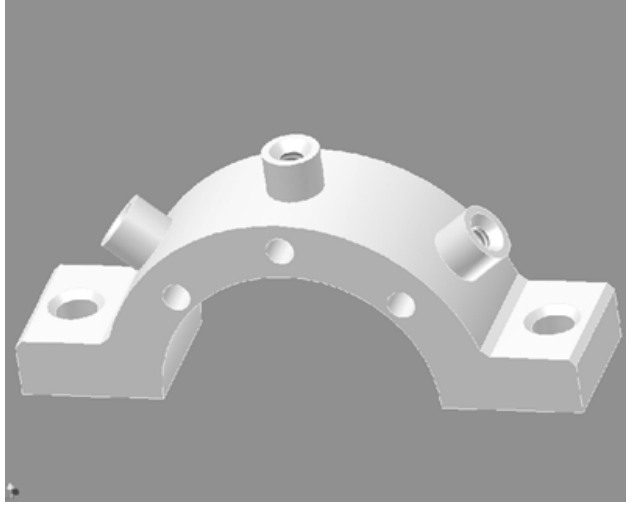

Fig. 3 Example of real technical body with the internal cavity

\section{Theory of simulation}

The process of modelling is closely related to the simulation. Simulation can be understood as process of executing the model. Simulation enables representation of the modelled real system or real process and its behaviour in real time by means of computer. The simulation enables also visualization and editing of the model.

A typical simulation model can be written both through specialized programming languages that were designed specifically for the requirements of simulations, or the simulation model can be created in standard programming languages, spreadsheets (MS Excel) or other application (CAD systems). From the above considerations, it is clear that simulation is a process that runs on the computer. In some publications, therefore, is called "computer simulation". It generally is valid that computer simulation is a computer-implemented method used for exploring, testing and analysis of properties of the conceptual (mathematical or process) models. That describe of the real systems or real process which cannot be solved using standard analytical tools [4].

The simulation models represented by executable computer program have to be isomorphic with the conceptual model that is a representation. It means that the mathematical model and simulation model have to represent the real system, its elements, internal interactions and external interaction with the environment in the same way. In our paper the parametric simulation realized in CAD application will be presented.

Significant function of the simulation

Simulation has from the scientific point of view several functions [5]. We will focus in this paper two of them and they are:

- Replacing the real process;

- Development of study process.

This is an important and indispensable feature of simulations and simulation model because it allows realize a situation of the process that cannot be investigated conventionally.

Computer simulation of real systems and real processes is one of the most effective methodological tools, especially for the following reasons:

Computer simulation of real systems and real processes is one of the most effective methodological tools, especially for the following reasons:

- It allows study models of reality that are not analytically solvable models, especially dynamic. 
data.

- Process and allows qualitatively and quantitatively classify a large amount of experimental

- Allows general theoretical models of experimentally confirm or disprove - validate.

\section{Visualization}

The concept of visualization is closely related to the concept of simulation. Visualization is a technique that transforms, selects and shows the output data and data obtained from the simulation allows the investigation, analysis and interpretation. Among the commonly used tools in scientific visualization, simulated data are 2D and 3D charts, tables, flowcharts, animated diagrams and more.

The main advantage of simulations is that simulations model allows providing rather big number of the process steps in relatively short time, changing of input parameters and its visualization and optimization of the process. The simulation and visualization is very useful from study point of view. Using the simulation model and visualization of simulation results on the screen, students can better understand the basic features of the processes and systems and develop their intuition. It is also essential that the teaching by means of simulation is much cheaper and faster than the teaching carried by real experiment. In some cases providing the real experiment cannot be feasible.

\section{Application of theory of modelling in parametric modelling}

Parametric modellers may use different environments and user interfaces, but the general principles of parametric modelling are similar. The relevant software application is mostly used as another creative tool. Models are created through sketching and through volume or spatial constructional elements. They must be clearly defined, without any redundant or contradictory information. There are always several solutions. Students must be able to deal with difficult and demanding logical tasks and must be able to use spatial imagination. This offers many opportunities for the application of alternative teaching methods, problem and project teaching techniques and the development of creative teamwork. The art of learning to solve problems lies in the fact that the student acquires the ability to independently seek solutions and strategies and to make decisions in alternative situations. Information technologies offer many opportunities for the application of alternative teaching methods, such as the problem and project teaching techniques and the development of creative teamwork. In order to strengthen cross-curricular relations, the Secondary School of Applied Cybernetics in Hradec Kralove introduced project teaching classes. One of the projects realized by the school is called the Virtual tour of objects. The technology of virtual reality is based on various fields such as programming, computer graphics, mathematics, but also on various artistic fields. Therefore, the above-mentioned project involves students focused on these fields of study. Students of the Computer Graphics class undergo classical art training and courses, which teach them the basics of computer graphic technology. Participants in the project are organized into two groups including future experts of various disciplines. Some are technically oriented (e.g. programmers) and others are artists. To achieve creative cooperation between these groups of students is not always an easy task.

\section{Research of the use of tools in the education of technician}

Progress of project work can be assessed both in the classroom observation, and by analysis of ongoing status of projects that listeners process outside school lessons. Final outcomes of completed projects can be also analyzed. The structure of CAx data enables to assess the level of technical thinking of a student, his spatial imagination and creativity. From this point of view, the most suitable are CAx applications that allow you to achieve the same result in different ways. Due to the materials possibilities of schools and to different licensing strategies for different providers of these systems, this requirement is difficult to reach.

Despite this fact CAx data created in any application are a carrier of sufficient amount of 
information to produce partial conclusions and for subsequent optimization of the concept of teaching, including teaching supports in the form of text and animated electronic materials. Animated materials are created directly through animation and visualisation tools of a used CAx application. They contain procedures of $2 \mathrm{D}$ and $3 \mathrm{D}$ design and setting up of corresponding simulations. Increase of knowledge and skills is verified by an experiment on two parallel groups in education. Sub-thematic unit is in the experimental group demonstrated with a use of a CAx tool. A classical method without the use of CAx applications is used in the check group. In both groups there are the same initial knowledge and skills, as measured by a pre-test. A post-test is entered and evaluated in both groups at the end of the learning process and this post-test is also repeated in a period of 1 month after completion of the interpretation of the topic. The results of the first post-tests of three final experiments are shown on figure 4. In all cases it was a demonstration of the cutting tool geometry using a CAD model. Several so specified experiments were carried out throughout the research. Selection of pupils to groups was made according to organizational options and criteria, which do not affect the examined parameters. Independence of choice was assessed on the basis of pre-test results. Numbers of pupils in each group were 15 to 30. An experiment was performed repeatedly with an emphasis on ensuring of the same conditions, consisting of the initial knowledge of students, course work, testing and evaluation.

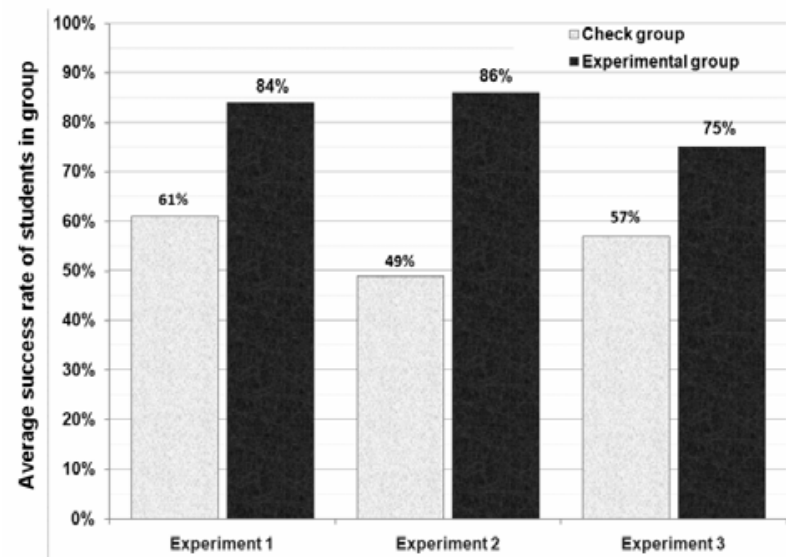

Fig. 4: Verification of deployment of a CAD tool in education - students' success in posttest.

Research of attitudes of students and graduates to CAx technologies and to the teaching supported by these instruments is carried out using a questionnaire and an interview. A web questionnaire for obtaining the necessary number of respondents also from remote locations was created for this purpose. Selected results of positive responses, which have a significant influence on the creation and optimization of conception of computer-aided education is presented in the graph on figure 5 .

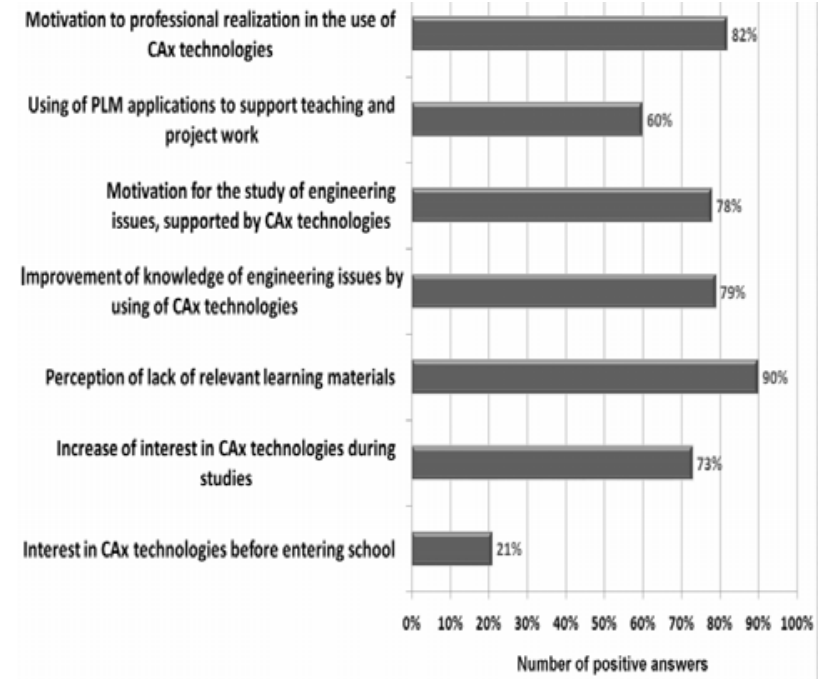

Fig. 5 Results of the survey of attitudes of students and graduates of technical schools to CAx technologies. 


\section{Conclusion}

The perception of visual information represents largest share of the entire perception done through all our senses. Visual presentation catches the attention, awakens interest and helps with conceptualization. Presentation together with practical demonstration is much more effective. Many terms and thoughts may be understood through visual methods rather than through verbal methods only, for example, practical skills. They should serve to help to expand the visual image. Therefore, it is necessary to keep in mind during the creation of a multimedia teaching educational application to add voice commentaries only to information that is not clear from the presentation itself. Regardless of the applied technology, it is clear that the creation of multimedia teaching supporting elements, and not only in connection with parametrical modeling, may be done by one person/teacher only and therefore it deserves a bigger share of our attention.

\section{Acknowledgment}

This article was created under the project called Specific research done at UHK Hradec Kralove no. 2115 in 2015 year.

\section{References}

[1] C. L. Sun, D. Y. Ning, W. Xiong, H. T. Wang, A Universal Feature Definition Frame for 3-D CAD Model Conversion, In: Advanced Materials Research, Vols. 690-693, pp. 2781-2786, 2013.

[2] M. Maněnová, V. Tauchmanová, Analysis of Communication Tools of the Learning Management Systems of Moodle and WebCt. In Education and educational technology (EDU'11). Athens: World scientific and engineering academy and society, s. 82-86, 2011.

[3] P. Vobornik, Mini-Language for Effective Definition of the Color Gradients, In: Advanced Materials Research, Vols. 1030-1032, pp. 1882-1885, 2014.

[4] S. Hubalovsky, Modeling and Simulation of Real Process - Passing through the Labyrinth as a Method of Development of Algorithm Thinking and Programming Skills. In: International journal of mathematics and computers in simulation. p. 125-133, 2013.

[5] W. Qiu, Study on the Normalization for Internet Computing and Modeling of Conceptual Model XML-Based, In: Advanced Materials Research, Vol. 121-122, 2010. 